\title{
Organisational Learning in SMEs: A Process Improvement Perspective
}

Purpose - We investigate how Organisational Learning (OL) can occur through Process Improvement (PI) activities and how this can lead to sustained improvement over time in the context of small and medium-sized enterprises (SMEs).

Design/methodology/approach - We study PI practices in six engineering-oriented SMEs via interview-based case studies. We draw from a range of literature and use an OL conceptual framework, informed by Crossan et al.'s (1999) 4I framework, as an analytical lens.

Findings - The OL perspective provides new insights to conceptualize the nature of PI as a multi-level practice in SMEs. Effective PI practices within SMEs are shown to be consistent with OL concepts, enabling firms to translate individually identified improvement opportunities into organisational-level changes resulting in sustained benefits. A new conceptual model is presented that explains how SMEs can learn though improvement activities. The key role of management support, both operational and strategic, is highlighted. It is necessary for management to provide sufficient PI opportunities to enable and sustain beneficial learning.

Research limitations/implication - The study is based on a sample of engineering-oriented SMEs located in the UK. Further case-based, longitudinal and survey-based research with firms of different types will enhance the generalisability of the findings, allowing the confirmation and extension of the new conceptual model.

Practical Implication - The findings provide a theoretically underpinned framework for OL in engineering-oriented SMEs deriving from PI activities. The new model highlights the key mechanisms that enable learning from improvement activities. The presented findings highlight the key role of management in introducing additional learning opportunities in the form of new business that requires exploratory learning. Without this, the reduction in improvement opportunities reduces the benefits that can be realised from PI.

Originality/value - OL provides a multi-level perspective to understanding how smaller firms are able to undergo systematic improvements and the support required to continually improve.

Keywords: Process Improvement; Organisational Learning; Continuous Improvement; SMEs; Theory. 


\section{Introduction}

Small and Medium-sized Enterprises (SMEs) are crucial in delivering sustainable growth in most economies (OECD 2014). Not only do SMEs represent the vast majority of firms (95\%) and employ the majority of the workforce (60-70\%) (OECD 2014), they are also an essential part of the supply chains of many larger firms (Söderberg and Bengtsson 2010). The ability of smaller firms to improve is therefore crucial - for their own survival, for the effective operation of many supply chains, and for the competitiveness of many economies (Chaston et al. 2001, Söderberg and Bengtsson 2010). However, SMEs face significant barriers when seeking to improve and develop. They are often hampered by limited access to necessary skills and resources, managerial competences and finance (OECD 2014).

Over the last three decades, there has been considerable interest in the concept of Organisational Learning (OL) to understand and explain how firms can change and create a competitive advantage over time (Fiol and Lyles 1985, Levitt and March 1988, Jones et al. 2010, Crossan et al. 2011). To improve competitiveness, attention is needed not only on individual level learning but also on how organisations can harness individual learning for organisational gain (Chaston et al. 2001). It is argued that organisations, particularly small ones, need to orient learning behaviours around specific organisational practices if they are to realize benefits from OL (Chaston et al. 2001, Altinay et al. 2015). However, while OL has been identified as an appropriate theory for use within operations management (Amundson 1998), it has been largely overlooked in contributing to theory development in the discipline (Boer et al. 2015, Walker et al. 2015).

Process Improvement (PI) and Continuous Improvement (CI) have been enduring themes across research and practice in operations management. PI and CI encompass a spectrum of activities, methods and approaches that seek to improve the effectiveness and efficiency of business processes over time and ensure the alignment of business processes with the competitive environment. PI and CI are fundamental in many formal organisational development and accreditation processes (Benner and Veloso 2008, Anand et al. 2009). They are of immediate and direct relevance to SMEs (Wolff and Pett 2006, Tidd and Bessant 2013). PI and CI may assist smaller firms in overcoming some of the limiting aspects associated with firm size (Wolff and Pett 2006). They have been identified as key mechanisms for improving SME productivity (Terziovski 2010). However, only a limited amount of research has been conducted on the detailed nature of improvement practices in smaller firms (Tidd and Bessant 2013). Research has discussed the relevance of established and influential improvement frameworks such as Six Sigma in the SME context (Antony et al. 2005, Kumar et al. 2006) but has not investigated the nature of improvement activities in such environments in detail. Research is needed to examine improvement practices in SMEs and how such practices relate to organisational level change (Chaston et al. 2001), in particular to understand how SMEs can learn through PI (Amundson 1998).

In this study we examine the role of PI and CI activities in providing a route to achieving beneficial learning at an organisational level in SMEs. We address two key issues - how OL occurs through PI/CI practices in SMEs and what OL factors ensure sustained benefits are 
realised from PI/CI in SMEs. The study is conducted with a sample of engineering-oriented SMEs, environments where improvement activities would be expected. For comparability, the companies studied all have ISO quality accreditations, a standard which emphasises the role of improvement activities (Benner and Veloso 2008). The study draws from the PI, CI and OL literatures to provide theoretical underpinning for the research and to provide a research framework to conduct the study. The research framework draws on the influential 4I OL framework (Crossan et al. 1999, Crossan et al. 2011) to analyse learning from PI/CI activities in the sample of SMEs.

The findings are presented in the form of a new conceptual model of learning through PI. Rather than the mere existence of particular practices mandated by established improvement frameworks, the study provides new insights to re-conceptualize the nature of improvement activities in SMEs as a multi-level combination of practices, behaviour and perceptions that can enable effective learning. The study highlights the key role of management in providing support and resources for PI activities and in generating new business that provides continuing opportunities for improvement initiatives. The analysis identified a group of companies able to achieve sustained benefits from improvement activities and a group of companies that were less effective at realising sustained benefits. In considering the findings, we argue that OL provides an appropriate and valuable perspective to understand and explain how SMEs can use PI to develop and meet the needs of their operating environment. The new conceptual model lays the ground for further empirical research to enhance understanding of how SMEs can use and support PI activities as a means to achieve OL.

The paper has seven subsequent sections. The next section discusses relevant literature and justifies OL as an appropriate theory to use to study PI in SMEs, leading to the formulation of the research questions. The qualitative research approach adopted is then discussed, including the selection of case companies and the methods used for data collection and analysis. The succeeding sections provide brief 'within case' and 'cross case' analyses. The findings are discussed in section 6 and a new conceptual model for organisational learning through process improvement in SMEs is presented in section 7. The paper concludes by discussing the relevance of the study to practice, contributions to theory, limitations and avenues for further research.

\section{Theory and Research Framework}

We consider the state of knowledge and understanding of Process Improvement (PI) and Continuous Improvement (CI), with particular attention given to the context of SMEs. We discuss limitations of previous research, arguing why OL provides a valuable lens from which to consider PI and CI. The provenance and use of specific OL frameworks is then discussed, providing a conceptual framework that connects OL with PI and CI. The research questions addressed in the study are then presented.

\subsection{PI and CI in SMEs}

PI has been highlighted as being central to operations management (Anand et al. 2010). CI is distinguished from PI in the literature by its emphasis on moving from isolated improvement 
activities to organisationally-driven approaches that encourage, support, and exploit such activities for improved performance over time (Bessant et al. 2001, Jørgensen et al. 2003, Anand et al. 2009). CI is viewed as an essential practice for firms to remain competitive in a dynamic business environment (Anand et al. 2009). Here we use the term PI/CI to reflect the spectrum of activities that occur in practice and the non-specificity of some of the research literature on process improvement.

Research on PI/CI in SMEs has focused principally on the refinement of existing processes to improve what is currently done (Wolff and Pett 2006, Terziovski 2010) through the application of previously developed tools and techniques, presenting the benefits firms are able to realize from them (Antony et al. 2005, Lo and Chang 2007). Antony (2001) and Kumar et al. (2006) illustrated how PI methods could be used to dramatically improve process performance using objective process data, but gave limited attention to the sustainability of improvements over time. The sustainability of PI efforts was taken as a focus of the work by Bateman (2005) who identified key inhibitors and enablers in realising benefits from PI activities and sustaining improvement activities over time.

The rigour and resources required by formal improvement approaches, such as Six Sigma, may not be wholly relevant for SMEs, who tend to favour less resource intensive approaches (Antony et al. 2005, Lo and Chang 2007). Benner and Veloso (2008) discussed how the implementation of ISO 9000 processes could support and enable deliberate changes of operational processes, arguing that the repeated use of formalised procedures supports the gradual improvement of performance through repetition. For CI, it is not only necessary to sustain improvements once they are made, but also to initiate follow-up PI activities (Bessant et al. 1994). Jørgensen et al. (2003) focused on CI and gave particular attention to the role of process review, which provides initiating points to identify and pursue operational PI. To explore how improvement activities can be sustained and become embedded in organisations, Jørgensen et al. (2008) examined the role of human resource practices to promote the engagement of operational staff and achieve CI. They illustrated how human resource infrastructure could formalise improvement practitioner roles, helping to embed improvement behaviours at an organisational level. Barton and Deldridge (2004) discussed how human resource practices could promote development at an individual level, that in time could create a competitive advantage. They also highlighted how individuals needed support in order for them to contribute to CI behaviours, due to discretionary effort acting as a potential inhibitor of CI efforts. Lam et al. (2015) explored this issue still further, highlighting the critical role managers play by providing behavioural support for promoting employee commitment within CI initiatives. This is consistent with the findings of Bateman (2005) who noted that personnel dedicated to PI activities promoted the sustainability of PI. Anand et al. (2009) discussed the role of formal infrastructure for achieving CI, not only for improving existing processes, but also for revising improvement systems to ensure they remain aligned with the external environment. Notwithstanding the need to embed improvement practices in order to achieve CI, Zangwill and Kantor (1998) noted how benefits from improvement activities could reduce over time as inefficiencies were removed from processes. 
Lee et al. (2000), Jørgensen et al. (2003) and Bateman (2005) identified the support and involvement of management, improvement goals, measures, and being provided with sufficient resources, as key enablers to sustain PI and achieve CI. Bessant and colleagues (Bessant et al. 1994, Bessant and Francis 1999, Bessant et al. 2001) identified similar topics of strategic leadership, tools and techniques, in addition to rewards and recognition. In particular, this stream of work suggested that firms could progress through five levels of CI maturity, each of which allowed greater benefits to be realised. The levels begin with random problem solving (level 0), through strategically aligned CI (level 3), to ultimately becoming a learning organisation (level 5). The model places attention on the development of CI infrastructure rather than focusing on operational activities, which may lessen its relevance to resource constrained SMEs. The empirical evidence gathered was primarily consistent with firms progressing to the strategic CI level (Bessant and Francis 1999), rather than achieving the hypothesised ideal of a learning organisation.

The literature distinguishes between the learning organisation and organisational learning (OL) - the former being much criticised (Tsang 1997, West and Burnes 2000). Garvin (1993) identified a selection of key practices necessary to build a learning organisation, which had parallels to enablers identified within the PI/CI literature (Bessant et al. 2001, Bateman 2005). He noted that "continuous improvement requires a commitment to learning" (Garvin 1993, p.78). Practices included management support, measurement, problem solving, working across organisational boundaries and incentives. However, while providing a framework to illustrate how improvement activities might be sustained, the learning organisation focuses on prescription, suggesting how an organisation should learn (Tsang 1997). Consequently, the concept of the learning organization provides insufficient insights to explain how organisations can and do actually learn.

There are gaps in the PI/CI literature on how improvement activities are carried out, specifically within small firms. Smaller firms tend to have a much greater focus on revenue generation (Terziovski 2010), with processes characterised by their informality (Marlow et al. 2010). Although representing a proportion of the firms involved in some previous studies on PI/CI (Bessant et al. 2001, Bateman and Rich 2003), attention was not given specifically to the requirements of SMEs. Even within work focused on improvement activities within smaller firms (Lee et al. 2000, Wolff and Pett 2006), attention has not been given to how frameworks or practices need to be adapted to account for a resource constrained context. Without such attention, suggestions related to formal, resource-based strategies, human resource policies (Bessant et al. 2001, Barton and Delbridge 2004) or improvement infrastructure (Jørgensen et al. 2003) may lessen their relevance for smaller firms.

Although previous research identifies a selection of practices to both sustain PI and enable the achievement of CI, the findings do not extend from the initial identification and testing of concepts to the building of relationships and theoretical frameworks for PI/CI or OL (Handfield and Melnyk 1998, Bryman 2012). Additionally, previous research has tended to give less emphasis to the details of operational PI, defining CI as "sustained incremental innovation" (Bessant and Francis 1999, p. 1107) without discussing what is being improved or how it results 
in sustained learning. Notwithstanding, PI has been identified as a primary source of innovation in small and medium-sized manufacturing firms (Terziovski 2010, Tidd and Bessant 2013) and therefore continues to have strong relevance for SMEs seeking to develop.

\subsection{Achieving OL through PI/CI}

In contrast to the 'learning organisation', organisational learning research seeks to understand the mechanisms that enable organisations to learn, change and adapt to account for the acquisition of new knowledge (Huber 1991). Building on previous reviews, Huber (1991) identified numerous perspectives on OL, from experiential learning related to practice, to vicarious learning from other individuals or organisations. Argyris and Schön (1992) explored issues at an individual and organisational level where gradual learning against established criteria could result in improvements in performance over time (defined as single loop learning). Within the CI literature, Zangwill and Kantor (1998) suggested that such forms of learning resulted in diminishing returns as system inefficiencies were removed. However, if a problem is more complex or different to existing frames of reference, individuals or organisations may be unable to resolve it. In such circumstances, Argyris and Schön (1992) argued that it was not only necessary for individuals or organisations to be able to solve problems based on existing criteria, but it was also necessary to engage in inquiry and to critically review existing mental frameworks (engage in double loop learning). This process not only allows organisations to improve activities they engage in, but also to exploit new opportunities and develop new processes and systems, similar to aspects of PI/CI (Jørgensen et al. 2003, Anand et al. 2009). Without engaging in such learning processes, organisations may, over time, become "brittle and unchangeable" (Argyris 1977, p.122). Levinthal and March (1993) provided considerable insights on issues associated with these two, potentially conflicting, forms of learning. While there may be a tendency to continually exploit existing mental frameworks to refine existing processes, they argued that the inefficiencies associated with exploration were less detrimental to long-term organisational health than overexploitation.

In addition to understanding individual learning processes (Argyris and Schön 1992), attention also needs to be given to how these processes relate to the organisation as a whole. Fiol and Lyles (1985, p.804) stated that "organizational learning is not simply the sum of each member's learning". Following the acceptance of new information, insight is developed that may result in improvements, which could be captured in organisational processes, systems, structures, and cultures, and affect subsequent behaviour. However, Fiol and Lyles (1985) also noted that an organisational crisis may be necessary to lead to the acceptance that established organisational processes and structures need to change. Nonaka and Takeuchi (1995) gave greater focus than previous research to the creation of knowledge from group activities, which link individuals with organisational level outcomes, to explain how learning could contribute to firm performance. They suggested that knowledge was created through conversion from a tacit state when held by individuals to an explicit state when codified. Engaging in these processes provided firms with key advantages by creating new knowledge and developing innovative solutions related to existing problems. In comparison to the other learning frameworks, Nonaka and Takeuchi (1995) gave attention to the forms of knowledge that may 
be difficult to codify (tacit knowledge). Although highly influential, and contributing to some CI thinking (Jørgensen et al. 2003), and to operations management more generally (Anand et al. 2010), the knowledge creation perspective overlooks how knowledge is exploited at an organisational level (c.f. Fiol and Lyles 1985). Notwithstanding these limitations, the work provides a valuable contribution to the improvement literature by linking group activities with organisational benefits.

There is a dearth of research specifically exploring OL within SMEs. Lee et al. (2000) noted that the vast majority of works on OL had been conducted within large organisations and thus were limited on the practical aspects of OL that could be implemented in SMEs. Chaston et al.'s (2001) work focused primarily on applying previously developed OL frameworks in the context of SMEs, and sought to illustrate how a range of OL practices might relate to improved SME performance. They found that it was only once organisational competences had been developed from learning activities that performance improved in SMEs. They recommended that more qualitative studies were needed to investigate how SMEs engaged in OL. Jones et al. (2010) examined learning in owner-managed small firms, focusing on the external connections possessed by the owner/manager and highlighting the role they play in institutionalising externally acquired knowledge. The work illustrates key gaps in the OL literature on SMEs and highlights the need to explore the mechanisms that relate operational practices (PI) to organisational benefits.

Huber's (1991) work provided valuable insights on the processes of OL. The process is initiated by knowledge acquisition, which needs to be converted to information and distributed through the organisation before being interpreted by those receiving the information and stored in organisational memory to be applied in the future. This enables OL to support long-term firm survival through alignment with a firm's environment, the "ultimate criterion of organizational performance" (Fiol and Lyle 1985, p.308). Based on a synthesis and analysis of previous work, Crossan et al. (1999) conceptualised OL as a process that acquires and intuits knowledge at an individual level, creates further knowledge at a group level through interpretation, and captures it at an organisational level. This process was encapsulated in their 4I framework illustrated in Figure 1. It shows the relationships between different learning practices, with lower levels feeding ideas to be discussed and developed within group activities, to allow the development of existing systems. It also shows how organisational level resources, such as policies, procedures and culture, feedback to direct lower levels of learning to inform behaviours, showing similarities with and an explanation of the exploitation of existing processes and mental models explored by Argyris and Schön (1992).

Crossan et al. (1999) argued that through this process, organisations could achieve strategic renewal, ensuring continued alignment with a dynamic operating environment. While highly influential within management research generally (Crossan et al. 2011), the 4I framework has received only limited attention within operations management and has not been investigated in a PI/CI context. Bontis et al. (2002) tested the framework empirically, finding that the learning flows (feedback and feed forward) were as strongly related to performance, if not more so, than previously accumulated resources. A particularly relevant example was Jones and Macpherson 
(2006) who extended the framework to illustrate how external support from customers, suppliers or consultancies could facilitate strategic renewal in SMEs. However, their work did not explore how involvement impacted internal practices or the role of internal learning and improvement activities on organisational level outcomes.

**insert Figure 1 about here**

Figure 1: The 4I Framework (source: Crossan et al. 1999, p.532)

Amundson (1998, p.351) advocated for research that explored "how OL occurs through process improvement". Table 1 identifies the main practices of OL identified from the literature and relates them to the literature on PI/CI. Given the similarities and complementary nature of the concepts, studying PI/CI from an OL perspective is valid contextually (Boer et al. 2015). The concepts in Table 1 provide the basis for a research framework to study PI from an OL perspective. Furthermore, reflecting on the practices and behaviours identified within the literatures on both PI/CI and OL, Crossan et al.'s (1999) 4I framework provides an analytical lens to examine the mechanisms by which PI/CI practices in organisations may relate to one another to achieve learning. In this study, we use these concepts and the 4I framework to examine how SMEs engage in PI/CI and how OL can be achieved through PI/CI activities. In contrast to the maturity model (Bessant et al. 2001) that attempts to identify firms with idealised processes, the analysis framework seeks to examine how SMEs can acquire knowledge and assimilate and utilise learning through PI.

Table 1: OL Conceptual framework for PI/CI

**insert table 1 about here**

Drawing from the previously presented literature and the need for further research into OL in SMEs, we pose the following two research questions:

\section{RQ1: How does OL occur through PI/CI practices in SMEs?}

\section{RQ2: What are the key $O L$ factors that ensure sustained benefits are realized from PI/CI within SMEs?}

Reflecting recent literature in the field of OL (Ingvaldsen 2015), the first research question highlights a necessary practical context in which to conduct research on OL. The second research question extends the first by examining the mechanisms that support learning processes to ensure the continued realisation of benefits from improvement activities in SMEs. The following section presents the research methodology employed in the study and explains how the collected data were analysed to answer the research questions.

\section{Research Approach}

Previous research has employed a range of approaches to investigate PI and CI, drawing from both objective and more subjective data to explore the relevance of previously developed frameworks (for example Antony 2001, and Anand et al. 2009). Approaches taken by Lee et 
al. (2000), Bessant et al. (2001) and Jørgensen et al. (2003) included action research and selfassessment to investigate improvement activities. While self-assessment tools may provide quantitative measures to compare the capabilities of different firms, McCutcheon and Meredith (1993, p.244) warned that such measures may be "cloaked in objectivity" and needed to be considered carefully. Lasagni (2012, p.331) also noted issues arising from such forms of selfassessment in smaller firms, creating a "bias toward self-confident SMEs" over estimating their capabilities.

A qualitative research approach using case studies has been adopted for this study. MacCarthy et al. (2013, p.940) state that operations management "needs more good qualitative work" to explore the issues being experienced by practicing managers. In particular, an interpretive approach to case studies was adopted to examine the relationship between OL and $\mathrm{PI} / \mathrm{CI}$ in SMEs. Interpretive case studies are appropriate when depth of understanding related to organisational practices is sought (McCutcheon and Meredith 1993, Meredith 1998). An interpretive perspective supports the discovery and description of organisational practices, without the need to impose a pre-conceived external perspective on interviewees (Noke and Hughes 2010). The research approach does not provide interviewees with concepts or assessment frameworks (external perspectives) for practitioners to consider (Noke and Hughes 2010), such as Lee et al. (2000) and Jørgensen et al. (2003). Instead, it allows practitioners to discuss topics in their own terms, using their own concepts. Understanding and interpretation of these concepts is developed through processes of active discussions between the interviewee and interviewer (Radnor 2001). The interpretivist approach differs from a strictly positivist approach to case research (Radnor 2001), giving less emphasis to the use of objective measures whilst placing greater emphasis on the meaning inherent in the information conveyed and its implications. It allows valuable follow up discussions, teasing out issues of relevance to the research study. An interpretivist approach does not preclude the use of other sources of information and data, and encourages the use of secondary data sources, product catalogues, web sites, brochures, and promotional materials. These were also used to support the analysis in this study.

\subsection{Selection and overview of cases}

Emphasis was placed on maximizing the diversity of firms involved in the study whilst maintaining a degree of comparability to ensure the robustness of insights developed and to strengthen external validity (Yin 2009). The selection criteria required that case study firms (i) had less than 250 employees, (ii) engaged in engineering related processes, and (iii) had an accredited ISO9000 quality management system (QMS). Given the selection criteria, firms were defined as 'engineering-oriented SMEs'. Formalized, externally-audited procedures have been identified as helping to facilitate PI by systematically changing procedures and ensuring improvements are cumulative (Benner and Veloso 2008). Formal operating procedures also represent organisational artefacts that provide evidence of capturing and deploying learning (Fiol and Lyles 1985, Jones et al. 2010). Requiring firms in the sample to have an accredited QMS reduced the variance between firms to some degree while ensuring firms had the necessary infrastructure to conduct process improvement (Lo and Chang 2007). 
Firms were selected from an extensive practitioner-oriented company database designed to facilitate business-to-business interaction. Companies were contacted via post, email and telephone, in order to introduce the research and highlight the benefits of involvement principally that feedback would be given on their PI practices in comparison to similar organisations. Six firms agreed to be involved in the study that were all located in the Midlands region of the UK. Selecting all of them helped maximize diversity and minimize case selection bias whilst ensuring the feasibility of the study due to the volume of interview transcripts and number of companies (Eisenhardt 1989). Reasons given by firms that chose not to be involved in the research related to the challenges being experienced in the period the research was conducted, which was in the aftermath of the global financial crisis. Case details are summarized in Table 2.

Table 2: Case Firms Summary

**insert table 2 about here**

\subsection{Interview approach}

To answer the research questions, it was necessary to discuss in detail the nature of PI practices as perceived by practitioners in the context of their operating and market environment. In contrast to direct questions used in previous OL research such as "do you believe that an employee's ability to learn is the key to improvement?" (Yeung et al. 2007) or "What mechanisms supported OL?" (Zhang et al. 2006), questions in this study were oriented around eliciting operational stories (Radnor 2001) about and in relation to PI/CI and OL. The interpretive approach avoids the researcher using particular terms with which a practitioner may be unfamiliar. Instead it allows interviewees to reflect on their experience and describe them in terms with which they are familiar. However, it should be noted that each of these questions led to follow up discussions on topics of direct relevance to PI/CI in these organisations. The theoretical lens of OL was used in the analysis to interpret organisational practices rather than to direct data collection, so reducing bias. The approach is consistent with approaches adopted to explore OL in the context of specific organisational practices, such as customer feedback (Caemmerer and Wilson 2010), inter-organisational relationships (Jia and Lamming 2013).

The firm was taken as the unit of analysis, with multiple respondents enabling an embedded, multiple case design (Yin 2009). Interviews were targeted at those in upper management. The majority of interviews were conducted with Managing Directors (see Table 2). Bessant et al. (2001, p.74) noted that a major advantage of conducting research with smaller firms was that Directors were "aware of what is going on... [and are] involved in allocating resources", enabling such respondents to report effectively on company-wide practices. Where possible, interviews were conducted with operational level staff directly involved in PI activities to provide alternative perspectives, particularly within the larger SMEs in the study. Additional sources of data other than interviews were used, such as websites that included project case studies, machine lists, and details of products and services provided, with additional observational data gathered from site tours. While the research primarily drew from 
interview data, other sources of information, particularly observations, are important for validating interpretivist research, providing "interpretive renderings of sounder quality" (Radnor 2001, p.51) and helping to triangulate interview data. The overall approach adopted helped to reduce some of the subjectivity and bias associated with case study research (Voss et al. 2002). In total 20 interviews were conducted, each lasting between 1.5 and 3 hours. All interviews were recorded with the agreement of participants and all were transcribed verbatim.

Following the introduction of the topics being researched when making initial contact with each firm, the interviewees (particularly those individuals not initially contacted) were briefed on the aims of the research and the topics that would be covered in the interview. In initial discussions with participants, examples of PI activities, such as operator initiated ideas, responses to returns from customers and activities to allow the introduction of new work were identified as possible areas where PI was likely to occur, providing a foundation for discussions on the topics. This enabled subsequent discussions to explore how operational improvement activities related to organisational outcomes within discussions on particular examples of PI. With each firm possessing an accredited QMS, how PI related to formalised systems focused discussions on topics interviewees were familiar with. Drawing from literature on PI and CI, discussions were elicited by asking questions related to the resolution of operational issues (e.g. internal non-conformance or returns from customers), the introduction of new machinery, the introduction of new business and the role of the QMS in the company (see Appendix 1).

Being deliberately broad, these topics provided opportunities for interviewees to cover aspects of the research framework, such as group work, formal procedures, management support, training and responsibilities given to individual staff. Without being explicitly directed, it was possible for interviewees to emphasise topics they considered important, with follow-up questions from the interviewer assessing whether topics included in the framework but not covered were relevant to the experiences of the interviewees. Questions led to discussion on topics of direct relevance to PI/CI processes in these organisations. For instance a question on handling a return from a customer led to follow up discussions on involving operational staff in the problem resolution process, with further questioning related to third parties that may become involved in solving more complex problems. An advantage of the interpretivist approach is that it reduces bias that may emanate from asking leading questions (Kvale and Brinkman 2009, p.301). By allowing interviewees to reflect on previously conducted improvement activities, it was possible to understand what initiated follow-up improvement activities. Evidence was also collected in each of the cases related to organisational level changes that had taken place over a prolonged period of time.

\subsection{Data analysis}

Before the analysis of the data, the interview recordings were repeatedly listened to and compared with notes taken within the interviews to assist with the appropriate interpretation of interviewee responses before the coding process took place (Radnor 2001). Data was analysed using the qualitative analysis software NVivo9. This helped to categorize and order the case database that consisted of over 36 hours of recordings and over 800 pages of transcriptions, documents and notes. The transcriptions were initially coded in relation to PI practices, 
providing examples of PI activities within each firm. The OL conceptual framework (Table 1) was then used to direct and focus the analysis of the examples of PI, categorising them as taking place at different organisational levels, how the levels related to one another, and how these related to PI outcomes. While employing a defined coding approach to structure the analysis, the process remained open to allow the identification of emergent themes not included in the initial coding (Radnor 2001, Noke and Hughes 2010). As a result, the research was deductive in assessing whether PI practices and the achievement of CI could be better understood through the lens of OL. The research was also inductive in terms of discovering key factors affecting the ability to benefit from and sustain process improvement activities within an SME context. External validity was provided by the OL theoretical underpinning (Barratt et al. 2011, Boer et al. 2015), allowing the development of a context-specific conceptual model of OL and PI/CI in SMEs.

Company specific case reports discussing process improvement were returned to each company for validation, helping ensure internal validity (Yin 2009, Noke and Hughes 2010). The validation of within case analysis was further augmented through follow-up interviews. The 4 case companies who were able to provide further meetings (ENG1, INJECT1, INJECT2 and BUILD) allowed the findings to be discussed in a holistic manner and validate whether the findings were representative of practices carried out within their firms. Case specific results were analysed in terms of how practices discussed with each firm related to the research framework, thus linking process improvement and organisational learning.

Each individual case report was independently reviewed in depth by the research team for corroboration. Similarities and differences in practices in the case companies were identified. Explicit methods were used (see section 5 below and Appendix $2 \mathrm{a}$ and $2 \mathrm{~b}$ ) to appraise the case companies and place into two groups - those that had strong PI/CI processes that enabled sustained benefits and those that had more limited processes in place and were less effective at engaging in, and sustaining benefits from PI. From analysis of interviews on improvement activities carried out over a number of years, it was possible to appreciate if and how improvement activities were made over time and their effects on the firm. From analysis of completed and on-going PI it was possible to elicit the extent that each firm was able to achieve and sustain benefits.

The cases were further reviewed and analysed to identify additional emergent, aggregate themes related to PI/CI and OL in SMEs. These were discussed within the research team and compared and contrasted between the more and less effective improvers. Emergent themes were also reviewed in the context of the existing literature (Eisenhardt 1989). This process supported a balance between the inductive identification of new themes and ensuring the external validity of findings by relating themes to established theoretical concepts (Barratt et al. 2011). Table 3 summarises the chain of evidence between PI/CI related discussion topics, the deductive, theoretically underpinned OL coding framework (Table 1), and emergent aggregate themes (Yin 2009). Following the in depth analysis and comparison between the two identified groups, a new conceptual model was developed grounded in the empirical evidence provided by the cases and informed by organisational learning theory. The new model is 
proposed to explain how organisational learning can be achieved effectively through PI/CI activities.

The following section provides brief summaries of the case companies in order to outline the context of the research. Example excerpts of the individual case data are presented, with interpretations of the data and their relationships to the coding framework.

Table 3: Chain of Evidence

**insert table 3 about here**

\section{Case Background and Within Case Analysis}

\subsection{Engineering Manufacturer 1 (ENG1)}

ENG1 works in sheet metal fabrication, operating as a job shop that produces customer-defined parts for a range of industries including automotive, rail and heating. Formed in the 1960s, ENG1 had undergone a management buyout in the late 1990s, following which, attention had been given to updating machinery, developing human resource practices and implementing and developing procedures in the form of an accredited QMS. Table 4 provides indicative example quotes related to PI activities in ENG1, the purpose and role of such activities, and the organisational level at which they occurred. As with Tables 5 to 9 in this section, the table does not represent the totality of evidence on PI/CI and OL in this organisation. More detailed evidence is provided in the Tables 10, Appendix 2a, 2b and Appendix 3. In addition to involving staff in product and process development activities, attention was specifically given by management in ENG1 to introducing new business and new machinery.

Table 4: ENG1 Case Analysis

**insert table 4 about here**

\subsection{Engineering Manufacturer 2 (ENG2)}

ENG2 is a job shop manufacturer that has produced compressed plastic parts primarily for the oil and gas industry for over 30 years. Due to the growth of domestic oil extraction, ENG2 enjoyed consistent orders for profitable work. Individual operators produce products on specific machines, allowing processes to be adapted and refined by operators without updating procedures. Table 5 provides indicative example quotes related to the PI activities carried out within ENG2, the purpose and role of such activities, and the organisational level at which they occurred. Management in ENG2 were involved in the updating of formal procedures following customer feedback, but did not take an active role in supporting improvement activities that involved operational staff. While individual operators took some responsibility for improvements, they did not always follow procedures and tended to resist manager initiated changes to their practice.

Table 5: ENG2 Case Analysis

**insert table 5 about here**

\subsection{Injection Moulding 1 (INJECT1)}

INJECT1 is an independent UK-based subsidiary of a global corporation. It had originally focused on injection moulded audio and video products for the consumer market. The 
subsidiary was founded in the early 1980s and grew rapidly to meet large demand for their products. Following contraction of existing markets, INJECT1 moved into the automotive sector in 2000. Table 6 provides indicative example quotes related to the PI activities carried out within INJECT1, the purpose and role of such activities, and the organisational level at which they occurred. Although a QMS was in place, due to resource limitations and resistance of operational staff to adhere to procedures, it had not been maintained or developed. In combination with the impact of the global financial crisis on the car industry, INJECT1 experienced problems to the point where an external party, employed by a major customer, provided support in making improvements. From the evidence, it was evident that INJECT1 experienced persistent problems and had limited upfront involvement with clients, tending to acquire similar repeat business or previously developed work won from competitors.

Table 6: INJECT1 Case Analysis

**insert table 6 about here**

\subsection{Injection Moulding 2 (INJECT2)}

INJECT2 was formed in 1989 and developed from producing components for the doubleglazing industry to supplying complex injection moulded components and assemblies to a range of industries. Exploiting the relatively high margin products of the double-glazing industry, INJECT2 accumulated the necessary equipment and developed complementary capabilities to maintain, design and manufacture their own tooling. Table 7 provides indicative example quotes related to the PI activities carried out within INJECT2, the purpose and role of such activities, and the organisational level at which they occurred. Within these processes, management provided resources for engagement in group activities, and supported individuals in taking responsibility for the improvements they made, which were subsequently integrated through management support into formal procedures. The continued introduction of new work offered additional opportunities to engage in beneficial improvement activities.

Table 7: INJECT2 Case Analysis

**insert table 7 about here**

\subsection{Systems Integrator (SYSINT)}

Two engineers who had previously worked within a large control systems equipment manufacturer formed SYSINT in 2002. They had identified an opportunity to provide a better service in designing and delivering advanced manufacturing technologies (combinations of control and production equipment) to a range of 'blue chip' clients. A major element of each of their projects was the provision of bespoke software that integrated separately manufactured, modular production and automation equipment. Table 8 provides indicative example quotes related to the PI activities carried out within SYSINT, the purpose and role of such activities, and the organisational level at which they occurred. Continuing problems experienced in SYSINT related to the software-writing element of the work. A range of issues resulted in a lack of responsibility being taken by individual engineers in adhering to procedures and management not formally implementing software writing procedures. Even though resources 
were directed to PI, it was apparent that the organisation had failed to make deliberate changes to address issues to exploit improvement opportunities.

\section{Table 8: SYSINT Case Analysis}

**insert table 8 about here**

\subsection{The Building Contractor (BUILD)}

BUILD was set up in 2001 to design, engineer and manage construction projects in the commercial sector, including warehouses and distribution centres for national supermarkets. A diversification strategy was pursued in 2008 to reduce the risk of operating within a sector where projects were at times pursued speculatively with bank funding. The diversification strategy included moving into the public sector, which was identified as an area of growth (at the time), which required the implementation of an accredited QMS. Table 9 provides indicative example quotes related to the PI activities carried out within BUILD, the purpose and role of such activities, and the organisational level at which they occurred. PI/CI was a part of BUILD's strategy to develop formal operational processes that supported improved consistency. Through management support to adhere to and update operational procedures, combined with working closely with customers, suppliers, and open group-based problem solving activities, BUILD were able to develop new solutions and deliver them consistently.

Table 9: BUILD Case Analysis

**insert table 9 about here**

\section{Cross Case Analysis}

The six case companies provide a spectrum of engineering-oriented SMEs, ranging from INJECT1 and ENG2 that focused primarily on manufacturing to SYSINT and BUILD that emphasised the delivery of engineering services. BUILD and SYSINT engaged in only limited manufacturing processes compared to INJECT1 and INJECT2, each of which had clearly defined manufacturing processes using specialized tooling. Between these extremes were ENG1 and ENG2 with a balance of tangible production equipment and intangible knowledge and operator skills to provide physical products, as well as advice and services in the redesign of existing products. This range of firms has provided the foundation for the development of robust insights relevant to other engineering-oriented SMEs.

Consistent with the literature on PI, CI and OL, there were some broad similarities across the sample in some aspects of how firms engaged in improvement activities, from initial problem identification to adaptation of formal procedures, consistent with their quality system accreditation. Table 10 shows evidence of similarities across the firms on processes for managing problems reported by customers, which reflects the QMS in place and the requirements placed on them by demanding customers. Notwithstanding these similarities, the effectiveness of PI varied across the sample.

Table 10 Similar PI activities across the sample of companies

**insert table 10 about here** 
The effectiveness of process improvement in each of these organisations was assessed using the totality of evidence gained from the study. Effective improvers were identified as those that were not only deliberate and proactive in their engagement in improvement activities, but where the empirical evidence indicated that they also derived sustained benefits from improvement activities. Less effective improvers were identified as those that engaged only in ad hoc, reactive improvement activities and where the empirical evidence indicated that sustained benefits were not realised and/or where persistent problems remained. Following review of each of the cases, there was agreement across the research team on the partitioning of companies into two groups. The approach is consistent with the case study methods adopted by others, such as Lockett et al. (2014) in the context of sensemaking about organizational change in healthcare.

Three of the firms (ENG1, INJECT2 and BUILD) were classified as effective improvers that showed clear evidence of sustained benefits from improvement activities. Appendix 2a provides specific evidence on (i) how each of these firms engaged in improvement activities, (ii) the internal and external support and engagement that were evident in undertaking improvement activities, and (iii) the types of sustained benefits generated. In all three of the effective improver cases there was evidence of benefits generated from improvement activities resulting in better competitive positioning of these firms to meet the requirements of new forms of business.

Three of the firms (ENG2, INJECT1 and SYSINT) were classified as less effective improvers that showed only limited evidence of sustained benefits from improvement activities and that had clear recurring or persistent operational problems. Appendix $2 \mathrm{~b}$ provides evidence on clear deficiencies in how these firms failed to engage effectively in improvement activities, including evidence in different cases on the lack of management support for improvement activities, the limited engagement of relevant personnel in development activities that could enable improvement activities, and the types of outcomes experienced. In all three of the less effective improver cases there was evidence of improvement activities not being effective at resolving problems and a limited ability to seize opportunities to improve.

In summary, PI provided a mechanism for some systematic improvement in all six case organisations, allowing firms to account for changes in their operating environment and identified opportunities. However, due to a range of factors, not all firms were able to realize sustained benefits from PI or continually improve. 


\section{Discussion}

In this section we discuss the insights gained from the study on how OL can occur through $\mathrm{PI} / \mathrm{CI}$ activities. This builds upon the evidence presented in Appendices 2a, $2 \mathrm{~b}$ and 3 to explain how the more effective and the less effective improvers were able, or not, to realise OL through PI activities. The empirical validation provides evidence with theoretical underpinning for specific organisational mechanisms that help to identify and explain how OL occurs through PI/CI practices in SMEs (Research Question 1) and the factors that ensure sustained benefits are realized from PI/CI (Research Question 2). Building on the analysis and discussion, we propose a conceptual model that explains the achievement of OL through PI/CI in SMEs in section 7 .

The lack of formal, consistently used and accepted operational procedures within the less effective improvers resulted in individuals within these firms developing their own approaches to completing work. Whether setting up moulding machines (INJECT1), operating CNC (computer numerical control) lathes (ENG2) or writing software code (SYSINT), personal experience accumulated through trial and error was the primary driver of practice. While developing personal expertise is essential to carry out some complex tasks, it also meant that individuals tended to be less willing to deliberately change, learn new practices or follow procedures if they were different to their personal practice. Whether taking account of feedback from customers, responding to direction from management (ENG2), working with external sources (INJECT1), addressing recurrent issues (SYSINT) or exploiting insights from individual staff (ENG2), it was difficult for the less effective firms to make and sustain changes to practices. In contrast, individuals in ENG1, INJECT1 and BUILD accepted deliberate changes to practice resulting from feedback from management, customers or staff. This was helped by procedures being developed by individuals through group discussions, which allowed them to perceive procedure changes as resulting, in part, from their own learning and knowledge, and thus being more willing to accept them.

The way in which individuals perceived improvement activities, engaged in group discussions, and adhered to operational procedures, highlight further factors affecting the ability of firms to engage in and sustain PI. The positive effects of individual perceptions in the effective improvers and the negative effects of individual perceptions in the less effective improvers could be identified. Building on how personal experience had developed, INJECT1 and SYSINT both highlight how such individually established behaviours were difficult to change. In comparison, management within ENG1, INJECT2, and BUILD gave significant attention to justifying new approaches and encouraging individual staff to accept new practices. This supported individuals in 'unlearning' out-dated practices, changing their perceptions and accepting new modes of practice. This is consistent with the findings from the PI (Bateman and Rich 2003), CI (Jørgensen et al. 2003, Lam et al. 2015) and OL (Fiol and Lyles 1985) literatures that identified such factors as important barriers to learning and sustained improvement.

Management support played a key role in determining whether the use of procedures was supported and the time that was provided for improvement activities. ENG2, INJECT1 and 
SYSINT showed that without support in these areas, it was difficult to effectively engage in and sustain changes made from PI, resulting in these firms engaging in ad hoc forms of improvement. In comparison, ENG1, INJECT2 and BUILD carried out practices that were more strongly consistent with the conceptual framework. They were able to proactively and deliberately change in response to customer feedback, identified improvement opportunities, and in pursuing and developing new business. Drawing from OL theory, effective improvers developed understanding of problems and opportunities within group discussions (Nonaka and Takeuchi 1995), with insights then captured within organisational procedures to be deployed in the future (Fiol and Lyles 1985). Management support was necessary to ensure that once developed, individuals took responsibility for adhering to these procedures.

Consistent with the PI/CI literature, following the initial pursuit of improvement activities, there can be a diminishing return of benefits as inefficiencies are eliminated (Zangwill and Kantor 1998). Within the case studies, this occurred due to major problems being identified, resolved and not recurring, with subsequent issues being less severe, and hence providing fewer benefits when solved. This gives rise to a paradox: if operational problems are resolved effectively, there may be fewer opportunities to engage in PI. A key issue, therefore, is whether the quantity and scope of improvement opportunities reduce over time: e.g. whether following effective engagement in PI activities, "there is nowhere to go" (PE, ENG1). The use of improvement suggestion schemes has been proposed in the PI/CI literature to sustain improvement activities (Bessant and Francis 1999, Lee et al. 2000, Jørgensen et al. 2003), as well as the introduction of incentives (Jørgensen et al. 2003) and formal infrastructure (Jørgensen et al. 2008, Anand et al. 2009). Apart from a general profit-sharing policy in ENG1, neither formal suggestion schemes, nor improvement-based incentive schemes were used in the case companies. However, some of the firms were still able to continuously improve with beneficial effects on the organisation because of the way that PI was viewed and supported. ENG1, INJECT2 and BUILD pursued developments through training staff, introducing new business, new process technology and working with new suppliers, which had a considerable impact on the nature and outcomes of PI activities. These activities were justified not only in terms of direct business arguments (e.g. increasing turnover), but as necessities for developing organisational capabilities to reflect current and future market requirements.

By neither deliberately identifying new business opportunities nor introducing new types of work, ENG2, INJECT1 and SYSINT continued their established behaviours and practices. INJECT1 and SYSINT did not have sufficiently close involvement with clients to allow individual staff to apply their knowledge. This was highlighted by INJECT1 and SYSINT working with customer-developed tooling and customer-defined project specifications, respectively. In contrast, ENG1, INJECT2 and BUILD proactively made changes to customerdefined designs, drawing from employee understanding in addition to capabilities of their suppliers to augment internal resources. New types of work required these firms to adapt internal processes through PI activities, resulting in a requirement to generate new insights, which could then be captured in new organisational processes necessary for consistently delivering new business. In contrast, for ENG2 that did not systematically introduce new business, the over-refinement of operational processes led to individuals resisting change, 
creating problems when it was necessary to introduce new equipment. While there is a requirement to maintain existing business, ENG2, INJECT1 and SYSINT illustrate the risks associated with the over-exploitation of existing processes that limit strategic development, reflecting OL theory (Levinthal and March 1993).

The introduction of new business can thus be viewed as a form of strategic management support necessary to provide opportunities to continue to engage in and sustain benefits from PI. This has not attracted explicit attention within the PI/CI literature, which has viewed the development of new products and processes as taking place only at the highest levels of CI maturity (Bessant and Francis 1999). Underpinned by organisational learning theory (Crossan et al. 1999), the research provides fresh insights for the role of the introduction of new business in achieving CI and ultimately OL. However, there is a need to balance exploratory (new business) and exploitative (existing business) forms of learning to both develop new capabilities while generating revenue from existing capabilities. New business can therefore be viewed as a source of opportunities that can initiate improvement activities in SMEs, similar to problem solving and waste reduction activities that have been the typical focus of PI/CI (Zangwill and Kantor 1998, Jørgensen et al. 2003, Bateman 2005).

\section{Conceptual model of organizational learning through process improvement}

We answer our two research questions through a conceptual model (see Figure 2), which was developed to show how OL can occur through PI in SMEs using the evidence gained from the analysis of the cases studied. In the context of engineering-oriented SMEs, the proposed model captures both the mechanisms by which learning occurs through PI/CI practices (Research Question 1) and the factors that influence the effectiveness of such learning in achieving and sustaining organisational benefits (Research Question 2).

The model presented in Figure 2 shows six main effects. The arrows show the direction of each effect as explained below. Appendix 3 provides specific evidence from each of the sustained improvers of the existence of each of the effects delineated in the model. Appendix 3 also highlights specific evidence of the behaviours present or not within the non-sustained improvers related to each of the effects.

**insert figure 2 about here $* *$

Figure 2: Conceptual model for OL through PI in SMEs

1) Individual perceptions play a key role in determining individual PI behaviours, which themselves impact individual perceptions (Effects 1.1 and 1.2). Individuals in the more effective improvers were willing and able to reflect on their own practices, question established approaches and perceive new ways of working (Effect 1.1), and change behaviours (Effect 1.2) when necessary. Without an ability to perceive new ways of working, individuals within the less effective improvers tended to maintain their personal perceptions of how processes should 
work (Effect 1.1), resulting in an unwillingness or inability to engage in problem solving activities, reinforcing existing behaviours that emphasised trial and error (Effect 1.2).

2) Individuals are willing and able to contribute problem solving and process knowledge to groups, leading to the development of new procedures that are effective for the organisation (Effects 2.1 and 2.2). For SMEs to engage in relevant and effective PI, it is necessary for individuals to discuss their experience of operational issues in group settings. Individual perceptions and behaviours influence their willingness and ability to contribute to group level discussions and problem solving (Effect 2.1). Following the development of solutions within groups, solutions need to be captured in organisational processes (Effect 2.2). In the more effective improvers, individuals were willing and able to contribute personal knowledge to group problem solving in developing solutions and new processes. Individuals in the less effective improvers were less willing to share individually developed approaches to working (Effect 2.1) and the organisations tended not to have formal systems in place to capture solutions (Effect 2.2).

3) Management support is essential at all levels of process improvement and problemsolving (Effects 3.1 to 3.4). The requirement to engage in PI needs to be stipulated by management to become an established organisational behaviour and the resources necessary to enable PI need to be provided at all three levels - individual, group and organisation. These include supporting individuals (via training and time) to follow procedures (Effect 3.1), providing time and resources to develop solutions at the group level (Effect 3.2), and implementing, maintaining, and mandating the use of procedures at the organisational level (Effect 3.3). Individual perceptions of PI can also be influenced by direct management intervention to explain and justify changes or the need for changes and improvements (Effect 3.4). In the more effective improvers, management support was evident in all problem-solving activities, and included personal support to change individual perceptions of PI. In contrast, in the less effective improvers, management was not actively involved in engaging operational staff in the need to accept new procedures. Management in the more effective improvers also provided critical organisational level support (Effect 3.3) by introducing new types of business that provided additional opportunities to engage in group problem solving, that required the introduction and learning of new procedures and process techniques. Within the less effective improvers, this element of management support was largely missing.

4) Group problem solving affects perceptions of individuals (Effect 4). Involvement in group problem solving affects individual perceptions of solutions and PI activities, improving acceptance of solutions. When individuals are willing to engage and contribute to group activities, group interactions and discussions, they are able to develop new insights on improvement opportunities, building on the experience of others, which leads to changes in their perceptions. A lack of participation in, or acceptance of group activities results in individuals maintaining their existing perceptions. By engaging in group problem solving, individuals in the more effective improvers were able to develop their perceptions of solutions and PI activities by building on the experience of peers and managers, improving the likelihood of them accepting developed solutions. Within the less effective improvers, a lack of 
involvement and acceptance of group activities allowed the maintenance of existing individual perceptions.

5) Formal organisational procedures affect individual behaviour (Effect 5). Formal procedures, when adhered to, directly affect individual behaviour and become accepted behaviour determining how individuals engage in operational activities. Through the repeated use of procedures, codified knowledge becomes part of an individual's tacit knowledge, affecting their perceptions. In the more effective improvers, documented organisational procedures were integral to how individuals operated. The absence of formally supported organisational procedures in the less effective improvers resulted in individuals maintaining personal operational behaviours and approaches to PI.

6) OL through PI can result in both operational and strategic benefits (Effect 6). Operational benefits are expected from PI activities and these were evident across the case companies (Effect 6a). Strategic benefits support firms in securing repeat business, working with new customers and in changing internal processes to support on-going organisational change (Effect 6b). In the more effective improvers, deliberate changes to product designs and processes provided reductions in costs and lead times, leading to the introduction of additional development work with new and existing customers. The less effective improvers exhibited only limited ad hoc operational improvements and were unable to engage in long-term firm development through PI activities.

The proposed model provides new insights on how PI activities in SMEs take place and how they relate to organisational level outcomes. The model extends previous research on PI (Bateman 2005) and CI (Bessant et al. 2001, Jørgensen et al. 2003), by explaining the mechanisms through which PI practices, when supported, can result in beneficial organisational learning in SMEs. In particular, the findings and proposed model highlights the roles of individual perceptions, group activities, organisational procedures, and management support in facilitating effective process improvement. This new insight builds upon Barton and Deldridge's (2004) ideas of discretionary effort, with the need to support and justify to those involved in PI activities the importance of making an effort and engaging fully in such activities. The findings also build upon Lam et al. (2015) by illustrating of critical role of management in building employee commitment to improvement activities. By identifying a lack of opportunities and individual perceptions are barriers to OL in SMEs, the research is also able to make contributions to OL theory, in terms of how individual perceptions impact learning at different levels of the organisation (Crossan et al. 2011).

Our research provides evidence for the dynamic nature of learning through PI in the SME context across the three levels of OL identified by Crossan et al. (1999). From the identification or introduction of improvement opportunities at an individual level, through the achievement of shared understanding and the development of improved operational processes at both individual and group level, revised procedures can be implemented at an organisational level, which ultimately results in organisational benefits. This is consistent with the findings of Altinay et al. (2015), who found that OL enabled firm to be entrepreneurial by providing a process for translating opportunities to business level benefits. How the different levels relate 
to one another are consistent with the conceptual research framework and the feed-forward and feedback processes identified by Crossan et al. (1999). The nature of groups in SME learning processes often involves inter-organisational groups with customers and/or suppliers and is consistent with the findings of Jones and Macpherson (2006), reflecting the need for SMEs to extend their limited resources. Additionally, the critical role of management support is evident at all levels for effective organisational learning to occur.

Although management support for group activities is vital, there was little evidence of organisational procedures impacting directly on group level behaviours in our sample. The lack of such effects is consistent with what would be expected given the nature of SMEs that tend to focus their formalised operational procedures on revenue generating processes (Terziovski 2010). Many of the group activities resulted from the introduction of new business, interaction with customers or suppliers and the identification of problems. Group behaviours were more informal (Marlow et al. 2010) and hence were not directed by operational procedures. However, for larger organisations, with more formal organisational structures, it is likely that the feedback loop from the organisation to the group level would be more clearly evident in stipulating group-related organisational procedures and policies.

\section{Conclusions and Research Implications}

The research has studied a spectrum of engineering-oriented SMEs, ranging from those focused primarily on manufacturing to those with a greater emphasis on providing engineering services. Applying an organisational learning lens across the six cases has helped to explain how effective and sustained process improvement with learning can occur in SMEs. The study makes contributions to theory and practice of PI/CI and OL in SME contexts.

The research has used an appropriate theory of OL to structure, analyse, and interpret observations of practice. A new conceptual model is presented that enhances our understanding of the relationships between PI/CI activities, OL, and the benefits that may be realised in SMEs. The conceptual model provides contributions to PI/CI and OL theory by explaining how different elements of PI, some identified in previous literature, relate to one another and to organisational outcomes. The research develops existing theories on PI/CI, emphasising the multi-level nature of effective process improvement that can generate learning of benefit to organisations. It provides insights on how problems are solved, how knowledge is created, captured and embedded into organisational systems, and later deployed over time. It also highlights factors that act as barriers to OL, in particular how individual perceptions can act to block these multi-level processes. The findings emphasise the critical role that management support plays at every level - individual, group and organisational levels. It also emphasises the strategic support that management can provide to maintain improvement opportunities by introducing new business into the organisation that requires continual process development and improvement.

The research has implications for SMEs and for policy makers in supporting and accelerating the development of OL in smaller firms through PI activities. Most directly for practice, it highlights the need for PI/CI activities to be viewed and supported at three levels - 
individual, group and organisation. Managers must understand their critical role in providing support at each level and in particular their role in influencing individual perceptions by emphasising the value that the organisation places on improvement, the need for individuals to engage positively in PI, and the need to follow procedures that have been formally established. The research also sends a message to managers that, notwithstanding the challenges of engaging in new forms of business, it can have a beneficial organisational effect in generating improvement opportunities for individuals and groups, resulting in beneficial organisational learning.

The study provides significant new empirical evidence to support the findings but, as with any study of this type, there are limitations. Building upon our findings, further research could be conducted to develop appropriate measurement constructs for the identified mechanisms and factors, relevant to the SME context, to form the basis for survey research. These could test specific relationships or the mechanisms and structure of the presented conceptual model across a larger number of firms. Such research could also assess more objective measures of process improvement and the benefits realised, for example, the number of changes to operational procedures, new product introductions, level of investment in new machinery or profitability of work. Additionally, longitudinal studies, although time consuming and resource-intensive to conduct, can add rich insights to our current understanding, helping to further explore causal relationships and processes of organisational evolution that enhance learning. Such research may provide new insights into how individual perceptions of PI change over time, how individual insights are integrated in group discussions, and the processes of selecting which insights are captured in procedures. Notwithstanding these limitations, the findings presented in this research provide a foundation, structure, and motivation to conduct such studies, with the new conceptual model of OL providing a framework through which to interpret findings. 


\section{References:}

Altinay, Levent, Melih Madanoglu, Glauco De Vita, Huseyin Arasli, and Yuksel Ekinci. 2015. "The Interface between Organizational Learning Capability, Entrepreneurial Orientation, and SME Growth." Journal of Small Business Management 54 (3):pp. 871-891.

Amundson, S.D. 1998. "Relationships between theory-driven empirical research in operations management and other disciplines." Journal of Operations Management 16 (4):341359.

Anand, G., P.T. Ward, and M.V. Tatikonda. 2010. "Role of explicit and tacit knowledge in Six Sigma projects: An empirical examination of differential project success." Journal of Operations Management 28 (4):303-315.

Anand, G., P.T. Ward, M.V. Tatikonda, and D.A. Schilling. 2009. "Dynamic capabilities through continuous improvement infrastructure." Journal of Operations Management 27 (6):444-461.

Antony, J., M. Kumar, and C. N. Madu. 2005. "Six sigma in small- and medium-sized UK manufacturing enterprises: Some empirical observations." International Journal of Quality \& Reliability Management 22 (8):860-874.

Antony, Jiju. 2001. "Improving the manufacturing process quality using design of experiments: a case study." International Journal of Operations \& Production Management 21 $(5 / 6): 812-822$.

Argyris, C. 1977. "Double loop learning in organizations." Harvard Business Review 55 (5):115-125.

Argyris, Chris, and Donald A Schön. 1992. On organizational learning: Blackwell Cambridge, MA.

Barratt, M., T.Y. Choi, and M. Li. 2011. "Qualitative case studies in operations management: Trends, research outcomes, and future research implications." Journal of Operations Management 29 (4):329-342.

Barton, H., and R. Delbridge. 2004. "HRM in support of the learning factory: Evidence from the US and UK automotive components industries." The International Journal of Human Resource Management 15 (2):331-345.

Bateman, N. 2005. "Sustainability: the elusive element of process improvement." International Journal of Operations \& Production Management 25 (3):261-276.

Bateman, N., and N. Rich. 2003. "Companies' perceptions of inhibitors and enablers for process improvement activities." International Journal of Operations \& Production Management 23 (2):185-199.

Benner, M.J., and F. M. Veloso. 2008. "ISO 9000 practices and financial performance: A technology coherence perspective." Journal of Operations Management 26 (5):611629.

Bessant, J., S. Caffyn, J. Gilbert, R. Harding, and S. Webb. 1994. "Rediscovering continuous improvement." Technovation 14 (1):17-29.

Bessant, John, Sarah Caffyn, and Maeve Gallagher. 2001. "An evolutionary model of continuous improvement behaviour." Technovation 21 (2):67-77.

Bessant, John, and David Francis. 1999. "Developing strategic continuous improvement capability." International Journal of Operations \& Production Management 19:11061119.

Boer, H., M. Holweg, M. Kilduff, M. Pagell, R. Schmenner, and C. Voss. 2015. "Making a meaningful contribution to theory." International Journal of Operations \& Production Management 35 (9):1231-1252. doi: 10.1108/IJOPM-03-2015-0119. 
Bontis, N., M.M. Crossan, and J. Hulland. 2002. "Managing An Organizational Learning System By Aligning Stocks and Flows." Journal of Management Studies 39 (4):437469.

Bryman, A. 2012. Social Research Methods. 4th ed. Oxford: Oxford University Press.

Caemmerer, B., and A. Wilson. 2010. "Customer feedback mechanisms and organisational learning in service operations." International Journal of Operations \& Production Management 30 (3):288-311.

Chaston, I., B. Badger, T. Mangles, and E. Sadler-Smith. 2001. "Organisational learning style, competencies and learning systems in small, UK manufacturing firms." International Journal of Operations \& Production Management 21 (11):1417-1432.

Crossan, M.M., H.W. Lane, and R.E. White. 1999. "An organizational learning framework: from intuition to institution." Academy of Management Review 24 (3):522-537.

Crossan, M.M., C.C. Maurer, and R.E. White. 2011. "Reflections on the 2009 AMR Decade Award: Do We have a Theory of Organizational Learning?" Academy of Management Review 36 (3):446-460.

Eisenhardt, K.M. 1989. "Building Theory from Case Study Research." Academy of Management Review 14 (4):532-550.

Fiol, C.M., and M.A. Lyles. 1985. "Organizational learning." The Academy of Management Review 10 (4):803-813.

Garvin, D. A. 1993. "Building a learning organization." Harvard Business Review 71 (4):7891.

Handfield, Robert B., and Steven A. Melnyk. 1998. "The scientific theory-building process: a primer using the case of TQM." Journal of Operations Management 16 (4):321-339. doi: Doi: 10.1016/s0272-6963(98)00017-5.

Huber, G.P. 1991. "Organizational learning: The contributing processes and the literatures." Organization Science 2:88-115.

Ingvaldsen, J.A. 2015. "Organizational learning: Bringing the forces of production back in." Organization Studies 36 (4):423-444.

Jia, F., and R. Lamming. 2013. "Cultural adaptation in Chinese-Western supply chain partnerships: Dyadic learning in an international context." International Journal of Operations \& Production Management 33 (5):528-561.

Jones, O., and A. Macpherson. 2006. "Inter-Organizational Learning and Strategic Renewal in SMEs: Extending the 4I Framework." Long Range Planning 39 (2):155-175.

Jones, O., A. Macpherson, and R. Thorpe. 2010. "Learning in owner-managed small firms: Mediating artefacts and strategic space." Entrepreneurship and Regional Development 22 (7-8):649-673.

Jørgensen, F., H. Boer, and F. Gertsen. 2003. "Jump-starting continuous improvement through self-assessment." International Journal of Operations \& Production Management 23 (10):1260-1278.

Jørgensen, F., P. Hyland, and L. Busk Kofoed. 2008. "Examining the role of human resource management in continuous improvement." International Journal of Technology Management 42 (1-2):127-142.

Kumar, M., J. Antony, R.K. Singh, M.K. Tiwari, and D. Perry. 2006. "Implementing the Lean Sigma framework in an Indian SME: a case study." Production Planning \& Control 17 (4):407-423.

Kvale, Steiner, and Svend Brinkman. 2009. Interviews, Learning the Craft of Qualitative Research Interviewing. 2 ed. Thousand Oaks, CA: Sage Publications Inc.

Lam, Marco, Mark O'Donnell, and Dan Robertson. 2015. "Achieving employee commitment for continuous improvement initiatives." International Journal of Operations \& Production Management 35 (2):201-215. 
Lasagni, A. 2012. "How can external relationships enhance innovation in SMEs? New evidence for Europe*." Journal of Small Business Management 50 (2):310-339.

Lee, G., D. Bennett, and I. Oakes. 2000. "Technological and organisational change in small-to medium-sized manufacturing companies: a learning organisation perspective." International Journal of Operations \& Production Management 20 (5):549-572.

Levinthal, Daniel A., and James G. March. 1993. "The myopia of learning." Strategic Management Journal 14 (S2):95-112. doi: 10.1002/smj.4250141009.

Levitt, B., and J.G. March. 1988. "Organizational Learning." Annual Review of Sociology 14:319-340.

Lo, Liang Kang, and Dong Shang Chang. 2007. "The difference in the perceived benefits between firms that maintain ISO certification and those that do not." International Journal of Production Research 45 (8):1881 - 1897.

MacCarthy, B. L., M. Lewis, C. A. Voss, and R. Narasimhan. 2013. "The same old methodologies? Perspective on OM research in the post-lean age." International Journal of Operations \& Production Management 33 (7):934-956. doi: 10.1108/IJOPM-08-2013-0373.

Marlow, S., S. Taylor, and A. Thompson. 2010. "Informality and Formality in Medium-sized Companies: Contestation and Synchronization." British Journal of Management 21 (4):954-966.

McCutcheon, D.M., and J.R. Meredith. 1993. "Conducting case study research in operations management." Journal of Operations Management 11 (3):239-256. doi: 10.1016/02726963(93)90002-7.

Meredith, J. 1998. "Building operations management theories through case and field research." Journal of Operations Management 16 (4):441-454.

Noke, H., and M. Hughes. 2010. "Climbing the value chain: Strategies to create a new product development capability in mature SMEs." International Journal of Operations \& Production Management 30 (2):132-154.

Nonaka, I., and H. Takeuchi. 1995. The Knowledge-Creating Company. New York, NY: Oxford University Press, Inc.

OECD. 2014. Financing SMEs and Entrepreneurs: An OECD Scoreboard.

Radnor, H.A. 2001. Researching your professional practice: doing interpretive research, Doing qualitative research in educational settings. Buckingham: Open University Press.

Söderberg, L., and L. Bengtsson. 2010. "Supply chain management maturity and performance in SMEs." Operations Management Research 3 (1):90-97.

Terziovski, Milé. 2010. "Innovation practice and its performance implications in small and medium enterprises (SMEs) in the manufacturing sector: a resource-based view." Strategic Management Journal 31 (8):892-902. doi: 10.1002/smj.841.

Tidd, J., and J. Bessant. 2013. Managing Innovation: Integrating Technological Market and Organizational Change. 5th ed. Chichester: John Wiley \& Sons.

Tsang, Eric. 1997. "Organizational Learning and the Learning Organization: A Dichotomy Between Descriptive and Prescriptive Research." Human Relations 50 (1):73-89. doi: 10.1023/a:1016905516867.

Voss, C., N. Tsikriktsis, and M. Frohlich. 2002. "Case research in operations management." International Journal of Operations \& Production Management 22 (2):195-219.

Walker, H., D. Chicksand, Z. Radnor, and G. Watson. 2015. "Theoretical perspectives in operations management: an analysis of the literature." International Journal of Operations \& Production Management 35 (8):1182-1206. 
West, P., and B. Burnes. 2000. "Applying organizational learning: lessons from the automotive industry." International Journal of Operations \& Production Management 20 (10): 1236-1252.

Wolff, J.A., and T.L. Pett. 2006. "Small-Firm Performance: Modeling the Role of Product and Process Improvements*." Journal of Small Business Management 44 (2):268-284.

Yeung, Andy C. L., Kee-hung Lai, and Rachel W. Y. Yee. 2007. "Organizational learning, innovativeness, and organizational performance: a qualitative investigation." International Journal of Production Research 45 (11):2459-2477. doi: 10.1080/00207540601020460.

Yin, R.K. 2009. Case Study Research: Design and Methodology (Applied Social Research Methods). 4th ed. Thousand Oaks, CA: Sage Publications Inc.

Zangwill, Willard I., and Paul B. Kantor. 1998. "Toward a Theory of Continuous Improvement and the Learning Curve." Management Science 44 (7):910-920.

Zhang, M., A. Macpherson, and O. Jones. 2006. "Conceptualizing the learning process in SMEs: Improving innovation through external orientation." International Small Business Journal 24 (3):299-323.

\section{Acknowledgements}

The authors would like to thank the firms involved in the research, without their significant contributions of time and effort, the research would not have been possible. The authors would also very much like to thank the anonymous reviewers for their valuable comments on the paper, which have helped to enhance its clarity and contribution.

\section{Appendix}

\section{Appendix 1: Interview Protocol}

\section{Demographic}

- Could you describe the nature of your job, with details of the different activities it involves?

\section{General}

- What continuous improvements initiatives are you undertaking at the moment?

\section{Training}

- What training programmes do you have in place?

\section{Process Improvement}

- On receipt of a non-conforming, what activities take place to resolve them?

- Is there a structured approach to problem solving?

- Do you have time formally allocated to resolving issues and implementing identified improvement opportunities?

- Can you describe the quality control processes of some of the parts you produce?

- How is the performance of corrective actions measured?

\section{New Product Development}

- When introducing new business, what activities does this involve?

- To what extent are customers involved in this process? 
- To what extent are suppliers involved in this process?

\section{Process Review}

- How are new product developments and process improvements documented?

\section{General}

- What other forms of process improvements take place (including changes to production machinery)?

- What is the general perception about the continuous improvement program among employees?

- In relation to return on investment, customer satisfaction, number of defective parts, waste and other measures of performance that are important to you, how does process improvement affect firm performance?

\section{Appendix 2a:}

**insert Appendix 2a about here** Appendix 2b:

**insert Appendix 2b about here** Appendix 3:

**insert Appendix 3 about here** 